\title{
Carcinoid Heart Metastasis with Pericardial Effusion. A Rare Presentation
}

\author{
Maldonado Suarez Joseph*, M.D. and Segarra Torres Amaury, M.D.
}

Department of Internal Medicine Residency Program, La Concepcion Hospital San German, Puerto Rico, USA

*Corresponding author: Maldonado Suarez Joseph, M.D., Department of Internal Medicine Residency Program, La Concepcion Hospital San German, Puerto Rico, USA.
Received Date: September 13, 2021

Published Date: October 04, 2021

\section{Case Report}

Carcinoid tumors are rare neuroendocrine malignancies (1 in 100,000 incidence) commonly arising from intestinal enterochromaffin cells. They secrete vasoactive substances, e.g., Serotonin, which cause the triad of flushing, diarrhea, bronchospasm, and Carcinoid Heart Disease (CHD) [1], its hallmark being right heart valvular dysfunction. Rare manifestations of CHD include cardiac (carcinoid) metastasis and pericardial effusions. We present a case of Carcinoid Syndrome with CHD and the rare occurrence of cardiac metastasis and pericardial effusion [2-4].

A 56 year female with PMHx of HTN arrived at our ED with 10 days of generalized moderate abdominal pain associated with weight loss, frequent watery diarrhea and facial flushing. She denied fever, vomiting, GI bleeding, chest pain, SOB, trauma, restaurant visits or travels. She appeared acutely ill although AAOx3. Bedside vital signs were stable. Physical exam revealed facial erythema, clear lungs, cardiac RRR, a 2/6 holosystolic murmur at the LLS border radiating to apex, normal PMI, decreased bowel sounds, moderate tenderness to palpation in all 4 quadrants without tympani, distention, rebound tenderness, guarding or masses. Labs revealed high CRP, positive FIT and high GGT. CXR was normal. EKG showed NSR with low voltage. Infectious diarrhea and acute abdomen were ruled out. Abdominopelvic CT showed an enhancing

$1.1 \mathrm{~cm}$ mural nodule, a $2 \mathrm{~cm}$ soft tissue lesion and heterogeneous enhancing hepatic lesions concerning for a terminal ileum neoplasm. Chest CT showed no lung disease, normal heart size and a small pericardial effusion. Liver biopsy confirmed a welldifferentiated neuroendocrine tumor. Elevated serum serotonin, chromogranin A, Pro-BNP and high 24 HR Urine 5-HIAA were also obtained. Echocardiography confirmed a small pericardial effusion without tamponade, thickening of the anterior tricuspid leaflet, mild tricuspid regurgitation, mild right heart dilation and a circumscribed, homogenous, non-infiltrating $1.5 \times 1.5 \mathrm{~cm}$ right atrial mass above and separate from the tricuspid anterior leaflet. Our patient's symptoms improved with antidiarrheal medications and octreotide therapy and was discharged home with multidisciplinary follow-up OPD evaluations with IM, Oncology, Surgery and Cardiology services [5, 6] (Figure 1).

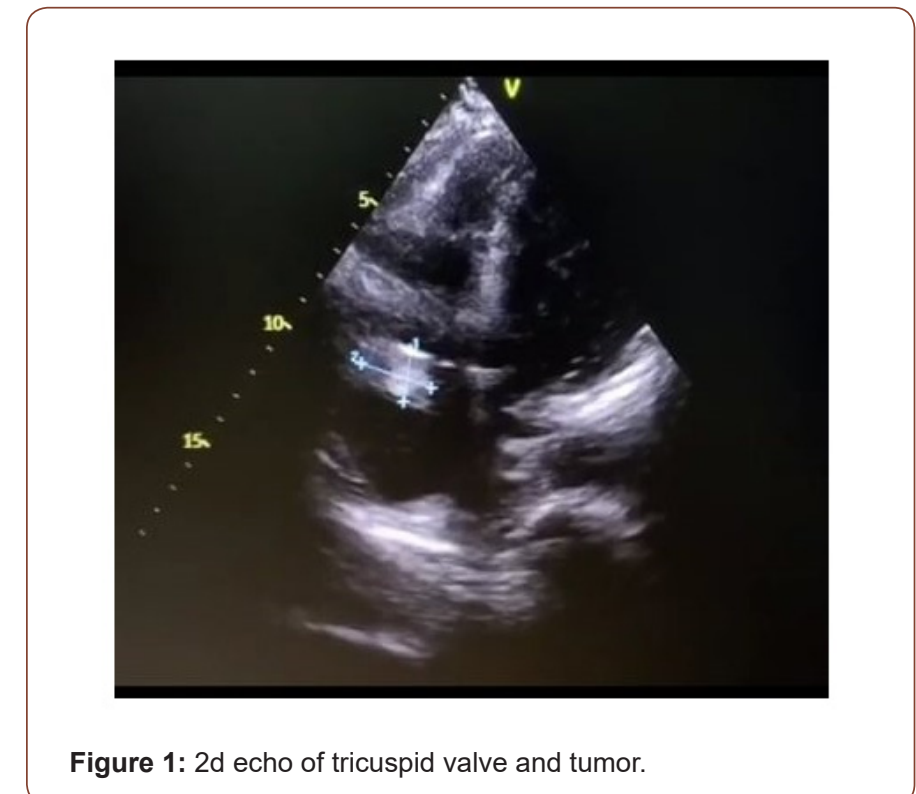

Carcinoid Heart Metastasis (4\% incidence) and pericardial effusions (14\% incidence) are rare complications of carcinoid tumor which may occur in the absence of carcinoid syndrome and 
CHD. Therefore, a high index of suspicion is needed. Complications include CHF, syncope, stroke, MI, pericarditis, tamponade, embolic events, arrhythmias, and death. Echocardiography can diagnose, monitor, and determine the timing for cardiovascular interventions. Somatostatin analogs, surgery of amenable cardiac lesions and tumor response to adjuvant therapies have led to symptomatic relief, functional improvement and improved quality of life. Therefore, we suggest echocardiography be performed in all patients with carcinoid tumor and a multidisciplinary approach to assure the best outcome. However further studies are needed to determine the impact of current therapies to overall survival of these patients $[7,8]$ (Figure 2).

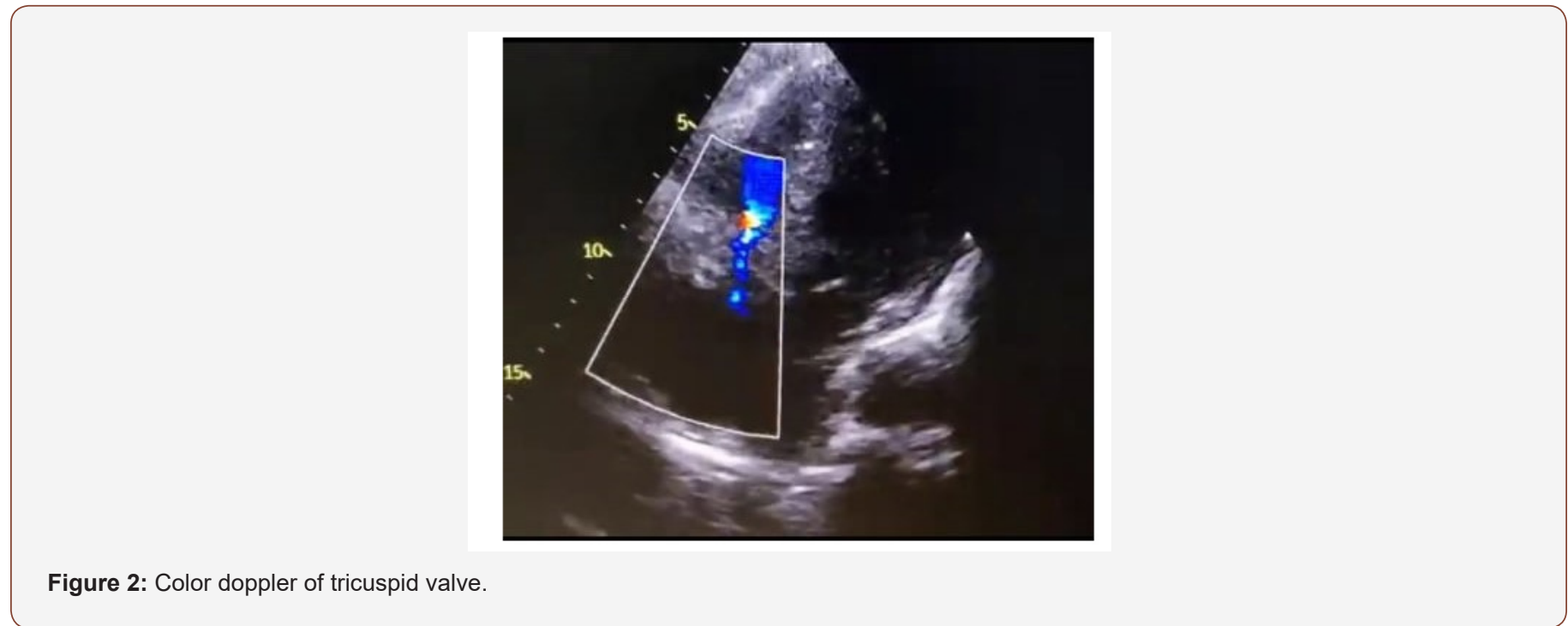

\section{Acknowledgement}

None.

\section{Conflict of Interest}

No conflict of interest.

\section{References}

1. Sanjeev Bhattacharyya, Joseph Davar, Gilles Dreyfus, Martyn E Caplin (2007) Carcinoid heart disease. Circulation 116: 2860-2865

2. Francesca Casadei, Antonella Moreo, Fancesco Musca, Paola Vallerio, Miriam Stucchi, etal. (2016) Cardiac involvement in carcinoid syndrome: Role of serial standard and contrast echocardiography. International Journal of Cancer and Clinical Research 3: 2378-3419.

3. Utpal H Pandya, Patricia A Pellikka, Maurice Enriquez-Sarano, William D Edwards, Hartzell V Schaff, et al. (2002) Metastatic carcinoid tumor to the heart: echocardiographic-patologic study of 11 patients. J Am Coll Cardiol 40: 1328-1332.
4. PA Pellikka, AJ Tajik, BK Khanderia, JB seward, JA Callahan, et al. (1993) Carcinoid heart disease. Clinical and echocardiographic spectrum in 74 patients. Circulation 87: 1188-1196.

5. Jon O Wee, Jerome D Sepic, Tomislav Mihaljevic, Lawrence H Cohn (2003) Metastatic carcinoid tumor of the heart. Annals of Thorasic Surgery 76: 1721-1722.

6. Gershan Davis, Kevin Birbeck, David Roberts, Naseer Naqvi (1996) Nonvalvular myocardial involvement in metastatic carcinoid disease. Postgrad Med J 72: 751-752.

7. Satish Sivasankaran, Anthony T Sonn, David M Venesy, Sherif B Labib, Bruce $S$ Tronic, et al. (2007) Metastatic cardiac carcinoid. Tex Heart Inst J 34: 132-133.

8. MH Hemnington, FC Detterbeck, MF Szwerc, ME Fidler (1997) Invasive catcinoid tumor of the heart. J Surg Oncol 66: 264-266 